Bull. Korean Math. Soc. 47 (2010), No. 6, pp. 1297-1310

DOI 10.4134/BKMS.2010.47.6.1297

\title{
TWISTED FACE-PAIRING 3-MANIFOLDS WHICH ARE HYPERBOLIC
}

\author{
Sungbok Hong And Kyunghwa Lee
}

\begin{abstract}
We construct a family of 3-balls using cones which represent closed orientable 3-manifolds and study twisted face-pairing construction due to Cannon, Floyd and Parry to understand the structure of such manifolds. Moreover, we prove that those manifolds are hyperbolic.
\end{abstract}

\section{Introduction}

The goal of this paper is to study the topological properties of certain closed orientable 3-manifolds obtained by twisted face-pairing construction. In [2], Cannon, Floyd and Parry established the basic properties of twisted facepairing manifolds. Besides, Cannon, Floyd and Parry investigated a special case of twisted face-pairing manifolds and the ample manifolds, and showed that these manifolds have Gromov hyperbolic fundamental groups in [3], they also showed how to construct Heegaard diagrams for twisted face-pairing 3manifolds in [4]. From their construction, it is easy to give framed surgery descriptions for twisted face-pairing 3-manifolds. In [5], they showed that the class of twisted face-pairing manifolds contains all lens spaces, the Heisenberg manifold (Nil geometry), $S^{2} \times S^{1}$, every orientable torus bundle over $S^{1}$ (Sol geometry), most closed, connected, orientable, Seifert fibered manifolds, and all connected sums of twisted face-pairing manifolds. It still seems unlikely that all closed, connected, orientable 3-manifolds can be obtained as face-pairing manifolds. It is shown that any twisted face-pairing 3-manifold that comes from an ample faceted 3-ball has a Gromove hyperbolic fundamental group in [4]. As a consequence they suggested the following question in [5].

Question. Is every twisted face-pairing 3-manifold that comes from an ample faceted 3-ball hyperbolic?

They considered twisted face-pairing manifolds which are hyperbolic and have small volume. Using SnapPea and the Dehn surgery description of twisted

Received May 7, 2009.

2000 Mathematics Subject Classification. 57M99.

Key words and phrases. twisted face-pairing, faceted 3-ball, twisted face-pairing 3manifolds, Takahashi manifolds. 
face-paring manifolds, they were able to construct hyperbolic manifolds with small volume. In Example 7.2 of [5], they introduced a hyperbolic manifold that comes from a model faceted 3 -ball $P$ identified with the tetrahedron by twisted face-pairing construction. Also, they applied twisted face-pairing construction to other model faceted 3 -ball $P$ such that $P$ is gotten from two tetrahedra by identifying a face of one with a face of the other ( $P$ is a hexahedron). In this case, they proved that the twisted face-pairing manifold with special multiplier is hyperbolic manifold of small volume in the Example 7.5 of [3].

In this paper, we obtain a partial solution for the above question. We first construct that the new model faceted 3-ball (cone faceted 3-ball) obtained from several tetrahedra by identifying a face of one with a face of the other. Also we show that, for all but finite number of the twisted face-pairing manifolds induced as above, the new faceted 3-ball are hyperbolic.

The main results of this paper are the followings.

Theorem 3.3. For any $n \geq 3$, let $M$ be a periodic Takahashi manifold given by $M_{n}\left(\frac{p}{q}, \frac{r}{s}\right)$. If $p=s$ and $r=-q=1$, then $M$ is a cone twisted face-pairing manifold $M_{n}(p)$.

Theorem 4.3. For any $n \geq 4$, the cone twisted face-pairing manifold $M_{n}(1)$ is hyperbolic.

This paper is organized as follows. In Section 2, we analyze the twisted face-pairing constructions, which are defined in [2], [3], [4], and [5]. Also we construct the cone twisted face-pairing 3-manifolds. In Section 3, we explain the terminology for twist moves on framed links in $S^{3}$, which are used throughout the paper. Most of these are from [6] and [9]. Also we introduce the periodic Takahashi manifolds. Furthermore, the connection between the periodic Takahashi manifold and the cone twisted face-pairing manifold will be discussed. In Section 4, using the topological structure of the periodic Takahashi manifolds, we show that most of cone twisted face-pairing manifolds are hyperbolic.

\section{Cone twisted face-paring construction}

\subsection{Twisted face-paring}

The twisted face-pairing construction is modeled on a faceted 3-ball. A faceted 3-ball $P$ is an oriented CW complex such that $|P|$ is a closed 3-ball, the interior of $P$ is the one open 3 -cell of $P$, and the cell structure of $\partial P$ does not consist of just one 0 -cell and one 2-cell. The face $f$ is gotten from a closed disk by identifying some vertices and some pairs of edges if the interior of $f$ is homeomorphic to the interior of a closed disk. Hence the such face $f$ admits faces of $\partial P$.

Now a face-pairing $\varepsilon$ on a given faceted 3-ball $P$ consists of the following. First, the faces of $P$ are paired: for every face $f$ of $P$ there exists a face $f^{-1} \neq f$ of $P$ such that $\left(f^{-1}\right)^{-1}=f$. Second, for every face $f$ of $P$, there exists a cellular 
homeomorphism $\varepsilon_{f}: f \rightarrow f^{-1}$ called a face-pairing map such that $\varepsilon_{f^{-1}}=\varepsilon_{f}{ }^{-1}$. We set $\varepsilon=\left\{\varepsilon_{f}^{ \pm 1} \mid f\right.$ is a face of $\left.P\right\}$.

Let $P$ be a faceted 3 -ball with orientation-reversing face-pairing $\varepsilon$ (A face pairing $\varepsilon$ is orientation reversing if every face-pairing map $\varepsilon_{f}$ reverses an orientation). Let $\sim$ be the equivalence relation on the set of edges of $P$ such that if $f$ is a face of $P$ and $e$ is an edge of $f$, then $e \sim \varepsilon_{f}(e)$. The equivalence classes of $\sim$ are called edge cycles.

$$
E: e_{1} \stackrel{\varepsilon_{f_{1}}^{ \pm 1}}{\longrightarrow} e_{2} \stackrel{\varepsilon_{f_{2}}^{ \pm 1}}{\longrightarrow} \cdots \stackrel{\varepsilon_{f_{n-1}}^{ \pm 1}}{\longrightarrow} e_{n} \stackrel{\varepsilon_{f_{n}}^{ \pm 1}}{\longrightarrow} e_{1} .
$$

Let $e_{1}, \ldots, e_{n}$ be the distinct edges of an edge cycle $E$ so that for every $i \in\{1, \ldots, n\}$ there exists a face $f_{i}$ of $P$ with $e_{i} \subseteq f_{i}$ and $e_{i+1}=\varepsilon_{f_{i}}\left(e_{i}\right)$, where the indices are taken modulo $j$.

For each edge cycle $E$, let $l_{E}$ be the number of edges in $E$ and we call $l_{E}$ the length of $E$. The function $m:\left\{\right.$ edge cycles $\rightarrow \mathbb{N}$ defined by $m(E)=m_{E}$ is called the multiplier function and a positive integer $m_{E}$ is called the multiplier of $E$.

Then we obtain a quotient space $P / \varepsilon$ consisting of orbits of points of $P$ under $\varepsilon$. We require that face-pairing maps satisfy the face-pairing compactibility condition from every edge cycle diagram.

$$
\left.\left.\left.\varepsilon_{f_{n}}\right|_{e_{n}} \circ \cdots \circ \varepsilon_{f_{2}}\right|_{e_{2}} \circ \varepsilon_{f_{1}}\right|_{e_{1}}=1_{e_{1}} \text {. }
$$

Hence we ensure that $P / \varepsilon$ is a cell complex.

Now we define a twisted face-pairing manifold from $P$ and $\varepsilon$. Let $Q=$ $Q(\varepsilon, m)$ be a faceted 3-ball from $P$ by just subdividing the edges of $P$ as follows. Let $e$ be an edge of $P$ and $E$ be the edge cycle of $e$. We subdivide $e$ into $l_{E} m_{E}$ subedges. The face pairing maps $\varepsilon_{f}$ on the faces on $P$ pairing maps on the faces of $Q$. For each face $f$ of $Q$, let $\tau$ be an orientation-preserving cellular homeomorphism of $f$ which takes each vertex of $f$ to its following vertex. Let $\delta$ be the face-pairing on $Q$ with the same pairing of faces as for the face-paring $\varepsilon$, and with $\delta_{f}=\varepsilon_{f} \circ \tau_{f}$ for each $f$ of $Q$. We set $\delta=\left\{\delta_{f}^{ \pm 1} \mid f\right.$ is a face of $\left.Q\right\}$. Then $\delta$ is a face-paring on $Q$ called the twisted face-pairing.

Define $M(\varepsilon, m)$ to be the quotient complex $Q / \delta$. The fundamental theorem of twisting face-pairing is that $M$ is a 3 -manifold. This was proved in Theorem 3.1 of [2]. We call $M(\varepsilon, m)$ a twisted face-pairing 3-manifold. For more details on these definitions see [2], [3], and [4].

\subsection{Cone twisted face-paring}

We will consider a special kind of face-pairing manifolds. We construct a model faceted 3-ball $P_{n}$ representing the closed orientable 3-manifold as follows:

\section{Step 1 : The cone faceted 3-ball.}

Let $P_{n}$ be a bi-pyramid with $n$-gon in $\mathbb{R}^{3}$. It is the union of two cones over a regular $n$-gon along a common face. We assume that $P_{n}$ is centered at the origin and its $n$ verties of valence 4 are in the $x y$-plane. The other two vertices 
are $n$-valence in $\mathbb{R}^{3}$. The vertices of the $p$-gon are denoted by $V_{1}, V_{2}, \ldots, V_{n}$, the vertices of the two cones by $N$ and $S$. We call $P_{n}$ a cone faceted 3-ball. See the Figure 1.

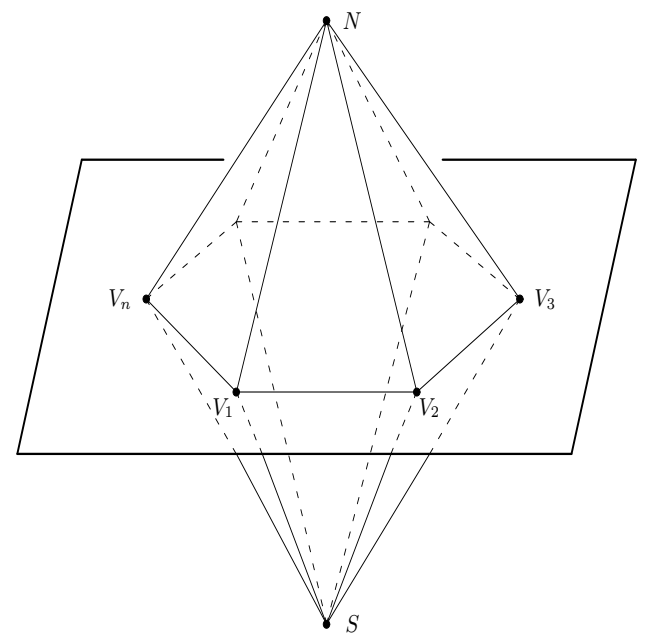

Figure 1. The cone faceted 3 -ball $P_{n}$

\section{Step 2 : The reflected face-pairing.}

Let $\varepsilon=\left\{\varepsilon_{i}^{ \pm 1} \mid i=1,2, \ldots, n\right\}$ be the orientation-reversing face-pairing map for which each face is paired with its image under reflection in the $x y$-plane and each face-pairing map is reflection in the $x y$-plane: For each $i$, we identify the face $f_{i}=\triangle V_{i} V_{i+1} N$ with the face $f_{i}^{-1}=\varepsilon_{i}\left(f_{i}\right)=\triangle V_{i} V_{i+1} S$, where the indices are taken modulo by $n$. We write using permutation notation in the following way:

$$
\varepsilon_{\boldsymbol{i}}=\left(\begin{array}{ccc}
V_{i} & V_{i+1} & N \\
V_{i} & V_{i+1} & S
\end{array}\right), i \in\{1, \ldots, n\} .
$$

In this case we call $\varepsilon$ reflected face-pairing. The edge cycles for $\varepsilon$ have $2 n$ diagrams as follows.

$$
\begin{aligned}
& E_{i}: V_{i} V_{i+1} \stackrel{\varepsilon_{i}}{\longrightarrow} V_{i} V_{i+1}(1 \leq i \leq n: \text { indices } \bmod n) . \\
& E_{j}: V_{j-n} N \stackrel{\varepsilon_{j-1}}{\longrightarrow} V_{j-n} S \stackrel{\varepsilon_{j-n}^{-1}}{\longrightarrow} V_{j-n} N(n+1 \leq j \leq 2 n: \text { indices } \bmod n) .
\end{aligned}
$$

Then $n$ edges in the $x y$-plane are in edge cycles $E_{i}$ of length $1, l_{E_{i}}=1(1 \leq i \leq$ $n)$, and each of the other $n$ edge cycles $E_{j}$ has length $l_{E_{j}}=2(n+1 \leq j \leq 2 n)$.

\section{Step 3 : Edge subdivision.}

Let $m_{E}$ be a multiplier function for a reflected face-pairing $\varepsilon$. We choose a multiplier function $m_{E}$ for each edge cycle so that positive integer $m=m_{E_{i}}=$ $m_{E_{j}}$ for $i \in\{1, \ldots, n\}$ and $j \in\{n+1, \ldots, 2 n\}$. Now, for each edge of edge 
cycle $E_{i}$, we subdivide edge into $l_{E_{i}} m_{E_{i}}$ subedges. So $n$ edges of $P_{n}$ in the $x y$-plane is subdivide into $l_{E_{i}} m_{E_{i}}=m(1 \leq i \leq n)$ edges in $Q_{n}$, and each of the other $2 n$ edges of $P_{n}$ is subdivided into $l_{E_{j}} m_{E_{j}}=2 m,(n+1 \leq j \leq 2 n)$ edges in $Q_{n}$. In our case each of the $2 n$ faces of $Q_{n}$ is a $5 m$-gon. See the Figure 2 .

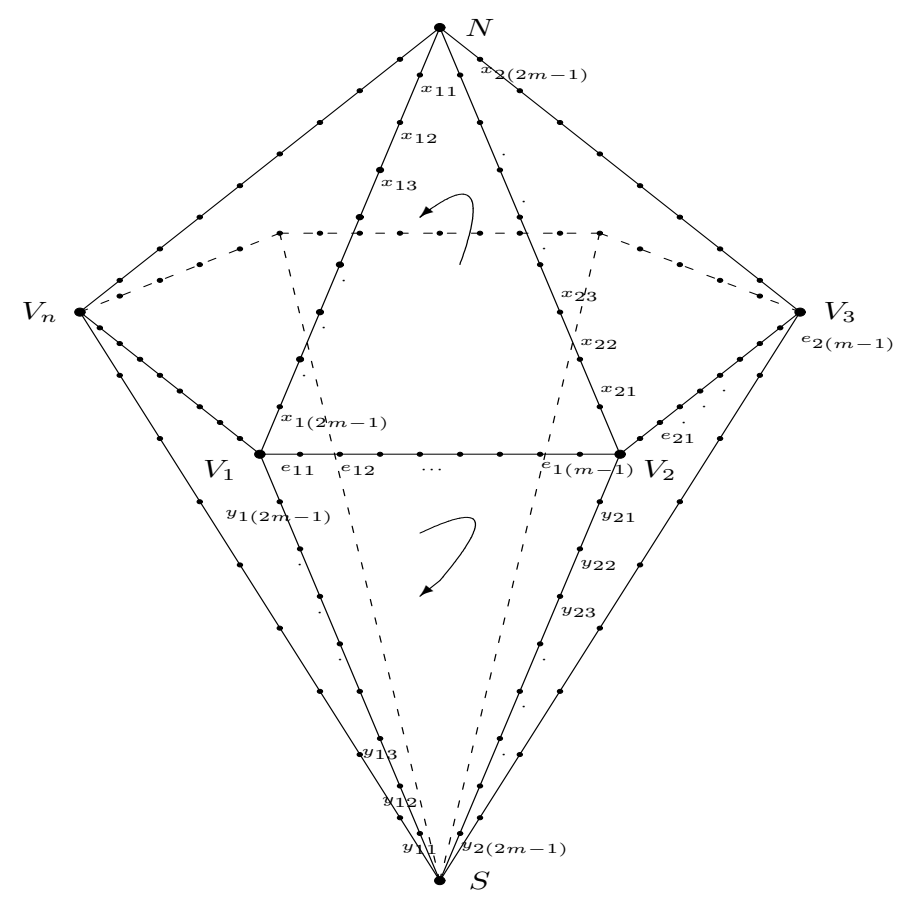

Figure 2. The complex $O_{n}$ for $P_{n}$

For example, $x_{11}$ is a new vertex in edge $V_{1} N$, and since the face-pairing map $\varepsilon_{1}$ takes $V_{1} N$ to $V_{1} S$, we have $\varepsilon_{1}\left(x_{11}\right)=y_{11}$. We now obtain

$\varepsilon_{i}=\left(\begin{array}{cccccc}V_{i} & e_{i 1} \cdots e_{i(2 m-1)} & V_{i+1} & x_{(i+1) 1} \cdots x_{(i+1)(2 m-1)} & N & x_{i 1} \cdots x_{i(2 m-1)} \\ V_{i} & e_{i 1} \cdots e_{i(2 m-1)} & V_{i+1} & y_{(i+1) 1} \cdots y_{(i+1)(2 m-1)} & S & y_{i 1} \cdots y_{i(2 m-1)}\end{array}\right), \quad(\bmod n)$.

\section{Step 4 : Cone twisted face-pairing.}

For our model we orient the boundary of every face of cone faceted 3-ball $P_{n}$ in the counter-clockwise direction. Before applying the induced face-pairing maps, we twist each face one subedge in the direction of the orientation of the boundary. Let $\tau_{i}$ be an orientation-preserving twisted cellular homeomorphism of each face of $P_{n}$ which takes each vertex of each face to its following vertex. 
Then, we can define $\delta_{i}=\varepsilon_{i} \circ \tau_{i}$. So we have

$\delta_{i}=\left(\begin{array}{cccccccccccc}V_{i} & e_{i 1} & \cdots & e_{i(2 m-1)} & V_{i+1} & x_{(i+1) 1} & \cdots & x_{(i+1)(2 m-1)} & N & x_{i 1} & \cdots & x_{i(2 m-1)} \\ e_{i 1} & e_{i 2} & \cdots & V_{i+1} & y_{(i+1) 1} & y_{(i+1) 2} & \cdots & S & y_{i 1} & y_{i 2} & \cdots & V_{i}\end{array}\right),(\bmod n)$.

Define $M_{n}(\varepsilon, m)$ be the quotient complex $Q_{n} / \delta$. We will denote $M_{n}(\varepsilon, m)$ by $M_{n}(m)$. One can shows that the cell complex $M_{n}(\varepsilon, m)$ is completely determined by $n$ and $m$ since, in our case, we fixed the face-pairing $\varepsilon$ by the reflection in the $x y$-plane.

Now we have the following theorem.

Theorem 2.1. Let $P_{n}$ be a cone faceted 3-ball with reflected face-pairing $\varepsilon$. Suppose given a multiplier function $m_{E_{i}}=m_{E_{j}}=m$ for $i \in\{1, \ldots, n\}, j \in$ $\{n+1, \ldots, 2 n\}$. Then the cell complex $M_{n}(m)=Q_{n} / \delta$ is an orientable closed 3-manifold.

Proof. Using the twisted face-paring construction, it is natural to construct the cell complex $M_{n}(m)$ from the cone faceted 3-ball $P_{n}$. Thus, by Theorem 3.1 in [2], the cell complex $M_{n}(m)$ is an orientable closed 3-manifold.

We call $M_{n}(m)$ a cone twisted face-pairing 3-manifold.

Now we compute the fundamental group of the cone twisted face-pairing manifold $M_{n}(m)$. As note above, we obtain the cycles of equivalent edges from cone twisted face-pairing $\delta$, as follows:

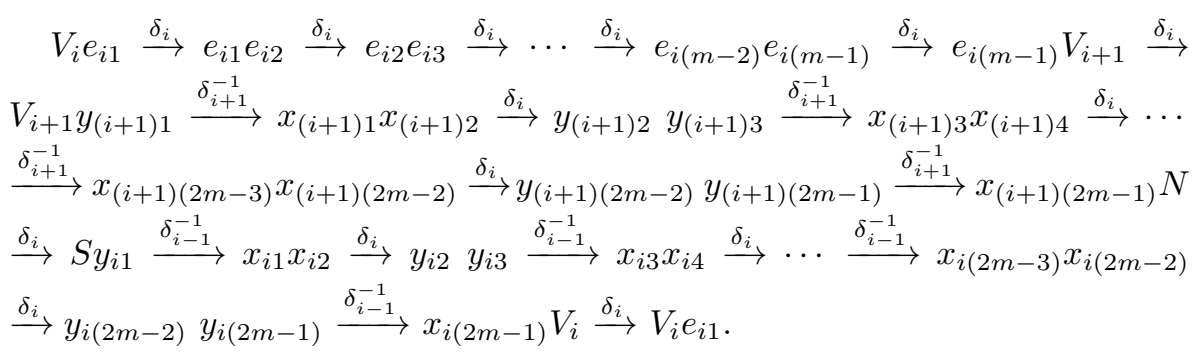

Let $\left\{x_{i}\right\}$ form a basis of a free group. We replace the $\delta_{i}$ atop the arrow in our edge cycle diagram by $x_{i}$. From our edge cycle diagram we give relations $x_{i}^{m}\left(x_{i} x_{i+1}^{-1}\right)^{m}\left(x_{i} x_{i-1}^{-1}\right)^{m}=1$ for any $i=1,2, \ldots, n(\bmod n)$. Thus we deduce the following theorem.

Theorem 2.2. For any $n \geq 3$, let $M_{n}(m)$ be a cone twisted face-pairing 3manifold. Then the fundamental group of the 3-manifold $M_{n}(m)$ admits the finite presentation

(1) $\pi_{1}\left(M_{n}(m)\right)=\left\langle x_{1}, x_{2}, \ldots, x_{n} \mid x_{i}^{m}\left(x_{i} x_{i+1}^{-1}\right)^{m}\left(x_{i} x_{i-1}^{-1}\right)^{m}=1: i=1, \ldots, n\right\rangle$,

where the subscripts are taken up to $\bmod n$. 


\section{The periodic Takahashi manifolds}

\subsection{Twists}

In this section we first review some well-known facts about the Dehn surgery. We focus on twist moves. This appears in Section 5.3 of [6] as Rolfsen twists and Section $9 . \mathrm{H}$ of [9]. Let $L=L_{1} \cup L_{2} \cup \cdots \cup L_{k}$ be a link $S^{3}$ framed by elements of $Q \cup\{\infty\}$. Suppose one component $L_{1}$ is unknotted. Then the complement $S^{3}-L_{1}$ of regular neighborhood of $L_{1}$ is a solid torus $T$. Let $t$ be a right hand Dehn twist of $T$ and $n \in \mathbb{Z}$. Let $L^{\prime}=L_{1}^{\prime} \cup L_{2}^{\prime} \cup \cdots \cup L_{k}^{\prime}$ be a new link obtained from $L$ by applying $t^{n}$ to $L-L_{1}$.

We frame $L^{\prime}$ as follows. If the first framing of $L_{1}$ is $r_{1}=\frac{b_{1}}{a_{1}}$, then the new framing of $L_{1}^{\prime}$ is $r_{1}^{\prime}=\left(n+\frac{1}{r_{1}}\right)^{-1}=\frac{b_{1}}{a_{1}+n b_{1}}$. If the first framing of $L_{2}$ is $r_{2}$, then the new framing of $L_{2}^{\prime}$ is $r_{2}^{\prime}=r_{2}+n\left[l k\left(L_{2}, L_{1}\right)\right]^{2}$, where $l k\left(L_{2}, L_{1}\right)$ is the linking number of $L_{1}$ and $L_{2}$, and similarly for $r_{3}^{\prime}$, etc. In [6] it is known that the manifold obtained by Dehn surgery on $L^{\prime}$ is homeomorphic to the manifold obtained by Dehn surgery on $L$.

\subsection{Periodic Takahashi manifolds}

In this section we prove that most of periodic Takahashi manifolds are cone twisted face-pairing manifolds.

We first define the Takahashi manifold. Let us denoted by $\mathcal{L}_{2 n}$ the $2 n$ component link of the Figure 3, which is a closed chain of $2 n$ unknotted components, with surgery coefficients $\frac{p_{1}}{q_{1}}, \frac{r_{1}}{s_{1}}, \ldots, \frac{p_{n}}{q_{n}}, \frac{r_{n}}{s_{n}} \in \mathbb{Q} \cup\{\infty\}=\tilde{\mathbb{Q}}$ cyclically associated to the components of $\mathcal{L}_{2 n}$, respectively. Takahashi manifold is closed orientable 3 -manifold obtained by Dehn surgery along the above link $\mathcal{L}_{2 n}$ (see [11] for more details). We denote $M\left(\frac{p_{1}}{q_{1}}, \ldots, \frac{p_{n}}{q_{n}} ; \frac{r_{1}}{s_{1}}, \ldots, \frac{r_{n}}{s_{n}}\right)$ by the Takahashi manifold determined by rational coefficients $\frac{p_{i}}{q_{i}}, \frac{r_{i}}{s_{i}}(i=1, \ldots, n)$ of $\mathcal{L}_{2 n}$. And we call $\mathcal{L}_{2 n}$ the Takahashi link. A Takahashi manifold is periodic when $\frac{p_{i}}{q_{i}}=\frac{p}{q}$ and $\frac{r_{i}}{s_{i}}=\frac{r}{s}$ for every $i,(i=1, \ldots, n)$. Denote by $M_{n}\left(\frac{p}{q}, \frac{r}{s}\right)$ the periodic Takahashi manifold. For details, see [8], [10], and [11]. From now on, without loss of generality, we assume that: $\operatorname{gcd}(p, q)=1, \operatorname{gcd}(r, s)=1$ and $p, r \geq 0$.

In [8], [10], and [11] they gave finite presentations of the fundamental group of the periodic Takahashi manifold $M_{n}\left(\frac{p}{q}, \frac{r}{s}\right)$. As a consequence, $\pi_{1}\left(M_{n}\left(\frac{p}{q}, \frac{r}{s}\right)\right)$ admits the following presentation with $2 n$ generators and $2 n$ relators:

$$
\pi_{1}\left(M_{n}\left(\frac{p}{q}, \frac{r}{s}\right)\right)=\left\langle x_{1}, \ldots, x_{2 n} \mid x_{2 i-1}^{q} x_{2 i}^{-r} x_{2 i+1}^{-q}=1, x_{2 i}^{s} x_{2 i+1}^{p} x_{2 i+2}^{-s}=1 ; i=1, \ldots, n\right\rangle,
$$

where the subscripts are taken up to $\bmod 2 n$. When $r=1$, we can easily get a cyclic presentation with $n$ generators:

(2) $\pi_{1}\left(M_{n}\left(\frac{p}{q}, \frac{1}{s}\right)\right)=\left\langle z_{1}, \ldots, z_{n} \mid z_{i}^{p}\left(z_{i}^{-q} z_{i+1}^{q}\right)^{s}\left(z_{i}^{-q} z_{i-1}^{q}\right)^{s}=1 ; i=1, \ldots, n\right\rangle$,

where the subscripts are taken up to $\bmod n$. 


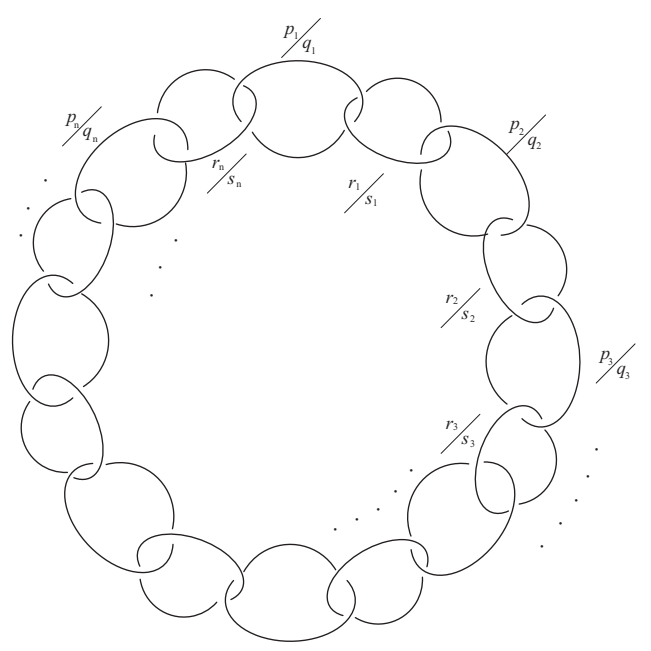

Figure 3. The Takahashi link $\mathcal{L}_{2 n}$

Lemma 3.1. For any $n \geq 3$, the fundamental group of the cone twisted facepairing manifold $M_{n}(m)$ is isomorphic to the fundamental group of the periodic Takahashi manifold $M_{n}\left(-m, \frac{1}{m}\right)$.

Proof. Using the equation (2), this follows easily from the equation (1) of Theorem 2.2. That is, if $p=s=m$ and $r=-q=1$, then we obtain the required presentation for the periodic Takahashi manifold.

The following lemma relates the link $L_{n}$ and the periodic Takahashi link, where $L_{n}$ is obtained by the corridor construction.

Lemma 3.2. For any $n \geq 3$, let $P_{n}$ be a cone faceted 3-ball with a multiplier function $m$. Let $D_{n}$ be a corridor complex link diagram for $P_{n}$. Let $L_{n}$ be a link in $S^{3}$ with diagram $D_{n}$. Define a framing of $L_{n}$ as follows. Every face component of $L_{n}$ has framing 0 . The edge components of $L_{n}$ corresponding to $E_{i}(i=1, \ldots, 2 n)$ has framing $\frac{1}{m}$. Then the link $L_{n}$ is isotopic to the periodic Takahashi link $\mathcal{L}_{2 n}$.

Proof. First of all, we construct $L_{n}$ as follows. As in Theorem 6.2.2 in [4], we can obtain the corridor complex link in Figure 4. A corridor complex for $P_{n}$ appears in Figure 4, drawn with thin arcs. A framed link diagram is drawn with thick arcs (circle).

A link component $L_{f}$ with framing 0 is presented for each triangular face in the northern cone. We call those components of $L_{n}$ as face components. Every edge $e$ of $P_{n}$ also gives a link component $L_{e}$ of $L_{n}$, called an edge component, as follows. Let $e$ be edge in the $x y$-plane of $P_{n}$. The edge cycle of $e$ is just $E_{i}(1 \leq i \leq n)$. From the argument in Section 2.2, we assign $L_{e}$ to be an 


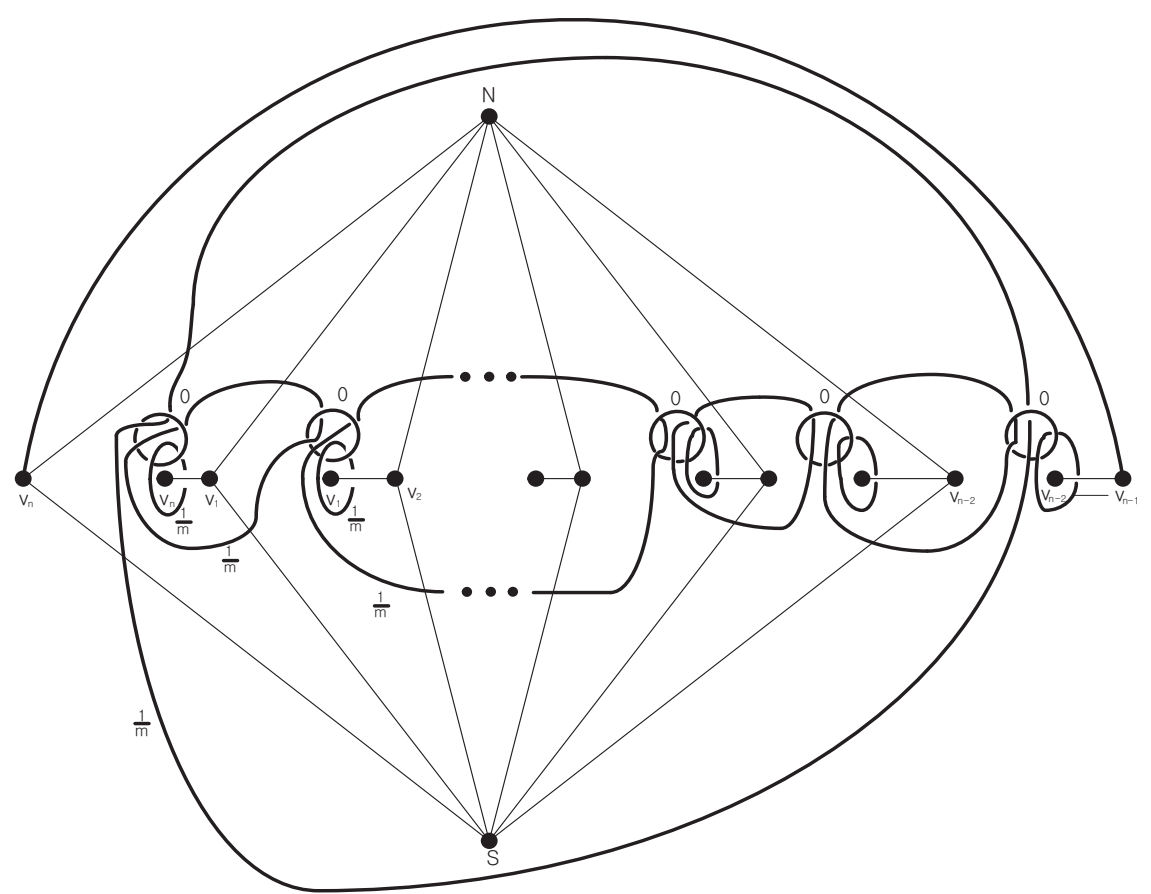

FiguRE 4. A corridor complex and framed link diagram for $P_{n}$

unknot with framing $\frac{1}{m_{E_{i}}}$, where $m_{E_{i}}=m$ is the multiplier of length 1 edge cycle. Now let $e$ be an edge of the northern cone not contained in the $x y$-plane. The edge cycle of $e$ is just $E_{j}(n+1 \leq j \leq 2 n)$. So we give $L_{e}$ to be an unknot with framing $\frac{1}{m_{E_{j}}}$, where $m_{E_{j}}=m$ is the multiplier of length 2 edge cycle. It is easy to see that the framed link in Figure 4 is isotopic to the framed link in Figure 5.

We simplify the component of the link $L_{n}$ in Figure 5 with framing $\frac{1}{m}$ by performing twist moves. Those components are made of the length 1 edge cycle. By twisting $-m$ times along those components with framing 0 , those components with framing $\frac{1}{m}$ change to components with $\infty$ framing. The same procedure changes components with 0 framing to components with $-m$ framing. By doing so, $L_{n}$ is isotopic to the link in Figure 6.

The link $L_{n}$ in Figure 6 is the periodic Takahashi link $\mathcal{L}_{2 n}$ with $2 n$-components.

Theorem 3.3. For any $n \geq 3$, let $M$ be a periodic Takahashi manifold given by $M_{n}\left(\frac{p}{q}, \frac{r}{s}\right)$. If $p=s$ and $r=-q=1$, then $M$ is a cone twisted face-pairing manifold $M_{n}(p)$. 


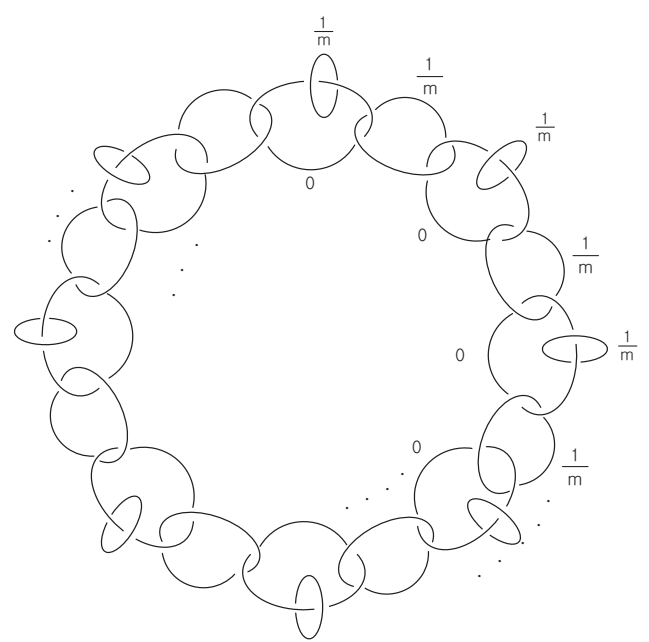

FIGURE 5. A simpler framed link $L_{n}$

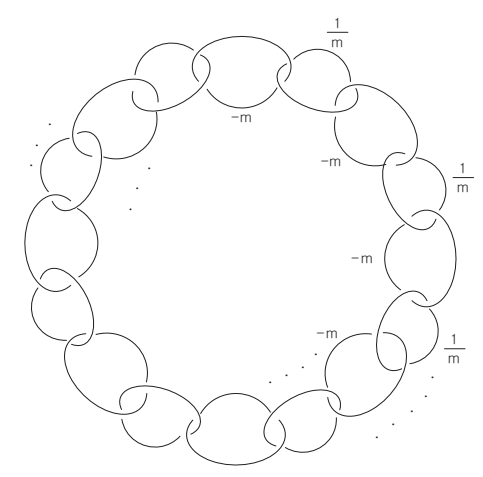

Figure 6. A simpler framed link $L_{n}$

Proof. Let $m$ be a positive integer such that $p=s=m$. The assumption implies that $\frac{p}{q}=-m$ and $\frac{r}{s}=\frac{1}{m}$. From the definition of the periodic Takahashi manifold, $M_{n}\left(-m, \frac{1}{m}\right)$ is obtained by Dehn surgery on $S^{3}$ along the periodic Takahashi link $\mathcal{L}_{2 n}$ in Figure 3. And Theorem 6.2.2 in [4] states that the associated cone twisted face-pairing manifold $M_{n}(m)$ is obtained by Dehn surgery on the framed link in Figure 6. Therefore Lemma 3.2 implies $M_{n}(m)$ is homeomorphic to $M_{n}\left(-m, \frac{1}{m}\right)$. 
From Theorem 3.3 we can deduce the following result, which has already been proved by Theorem 3 in [8].

Corollary 3.4. For any $n \geq 3$, the cone twisted face-pairing manifold $M_{n}(m)$ is the $n$-fold cyclic covering of the connected sum of lens space $L(m, 1)$, branched over a knot $K$, which does not depend on $n$.

\section{Hyperbolicity}

In this section we state some results about the hyperbolicity of the cone twisted face-pairing manifold $M_{n}(m)$. Also we give the main theorems.

For arbitrary faceted 3-ball $P$, we need to define an ample faceted 3-ball. This appear on p. 1 of [3].

Definition. A faceted 3-ball $P$ is ample if it satisfies the following conditions:

(i) every two distinct faces of faceted 3-ball are either disjoint or meet in a vertex or meet in an edge.

(ii) three distinct faces of a faceted 3-ball which meet each other pairwise have exactly one vertex in common.

(iii) no face of a face 3 -ball is a triangle.

Then these conditions are called the ampleness conditions.

We consider that a twisted face-pairing 3-manifold $M(\varepsilon, m)$ which is obtained by an ample faceted 3-ball $P$; such a 3 -manifold $M(\varepsilon, m)$ is called an ample twisted face-pairing manifold on $P$.

Theorem 4.1. Let $P_{n}$ be a cone faceted 3-ball, and let $m$ be a multiplier function. Let $M_{n}(m)$ be the associated cone twisted face-pairing manifold. Then $\pi_{1}\left(M_{n}(m)\right)$ is Gromov hyperbolic.

Proof. According to Theorem 6.1 of [3], it suffices to show that $M_{n}(m)$ is an ample twisted face-pairing manifold. We consider an ample faceted 3-ball since the ample twisted face-pairing manifold can be obtained by the ample faceted 3 -ball. We can construct an ample faceted 3-ball from our cone faceted 3-ball $P_{n}$ as follows. We perform the dual cap subdivision of each faces of $\partial P_{n}$, which means that every triangle face is subdivided as in Figure 7 . We denote by $P_{n}^{*}$ the subdivision 3 -ball for $P_{n}$. Then $P_{n}^{*}$ has ampleness conditions (i), (ii) and (iii).

Actually $P_{n}^{*}$ can be obtained from $P_{n}$ so that it is $\varepsilon$-invariant. So a reflected face-pairing $\varepsilon$ on $P_{n}$ naturally determines a face-pairing $\varepsilon^{*}$ on $P_{n}^{*}$. This completes the proof of Theorem 4.1 .

The following is the main theorems.

Theorem 4.2. For any $n \geq 4$, the cone twisted face-pairing manifold $M_{n}(1)$ is hyperbolic. 


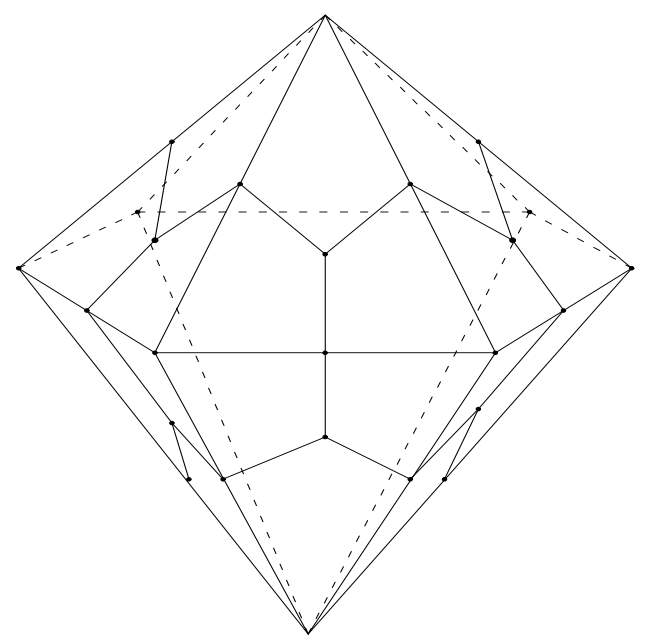

FIGURE 7. The ample faceted 3-ball $P_{n}^{*}$

Proof. By Theorem 3.3, we observe that, for $m=1$, cone twisted face-pairing manifold $M_{n}(1)$ is homeomorphic to $M_{n}(-1,1)$. On the other hand, the periodic Takahashi manifold $M_{n}(-1,1)$ is the Fibonacci manifold $M_{n}(1,-1)$ by the symmetry of the periodic Takahashi link $\mathcal{L}_{2 n}$ of Figure 3 (see p. 3 in [8] for more details). Then, for any $n \geq 4$, it was proved that these manifolds $M_{n}(1,-1)$ is hyperbolic in [7]. This completes the proof.

Proposition 4.3. For any $n \geq 3$, let $P_{n}$ be a cone faceted 3-ball with a multiplier function $m$. Let $D_{n}$ be a corrider complex link diagram for $P_{n}$. If $L_{n}$ is a link in $S^{3}$ with diagram $D_{n}$ (see Figure 5). Then the link $L_{n}$ is hyperbolic.

Proof. Lemma 3.2 implies that the corridor complex link $L_{n}$ (see Figure 5) is isotopic to the Takahashi link $\mathcal{L}_{2 n}$ of Figure 3. We simplify the Takahashi link $\mathcal{L}_{2 n}$ in Figure 3 using twist moves. Twisting $-m$ times along the each components with framing $\frac{1}{m}$, and deleting resulting components with framing $\infty$ yields the link of $n$-components of Figure 8 . The same procedure changes components with $-m$ framing to components with $-3 m$ framing. After these simplifications, one can deduce that the simplified Takahashi link $\mathcal{L}_{2 n}$ of Figure 8 is hyperbolic by using the result in [1] (see p. 222) and [12] (see p. 144). So the link $L_{n}$ is hyperbolic.

As a result of the above proposition, we can deduce the hyperbolicity for the cone twisted face-pairing manifold $M_{n}(m)$. By using the Thurston-Jorgensen's theorem for hyperbolic surgery which states that all but finitely many Dehn 


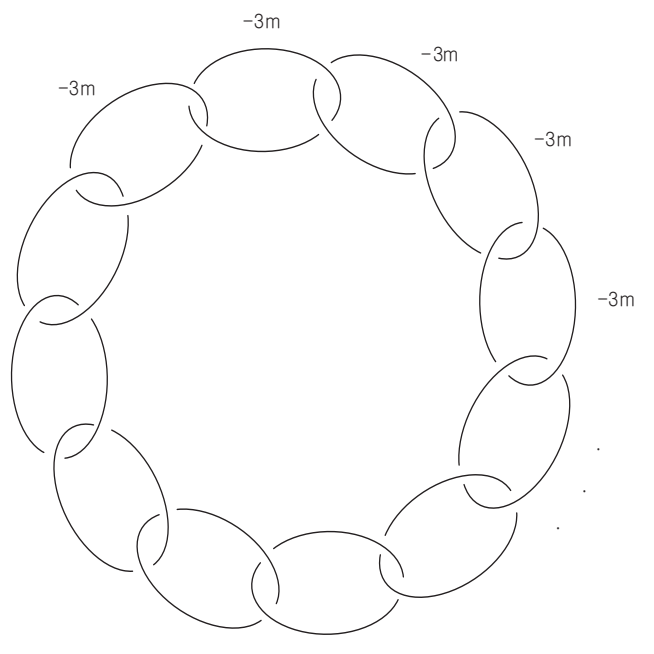

Figure 8. The simplified Takahashi link $\mathcal{L}_{2 n}$

surgery on hyperbolic link complements yield hyperbolic structure (see [12]), we obtain the following theorem.

Corollary 4.4. For any $n \geq 3$, and for all but finite number of multiplier $m=\left\{m_{i} \mid i=1, \ldots, n\right\}$, the cone twisted face-pairing manifolds $M_{n}(m)$ are hyperbolic.

\section{References}

[1] R. Benedetti and C. Petronio, Lectures on Hyperbolic Geometry, Springer-Verlag, BerlinHeidelberg-New York, 1992.

[2] J. W. Cannon, W. J. Floyd, and W. R. Parry, Twisted face-pairing 3-manifolds, Trans. Amer. Math. Soc. 354 (2002), no. 6, 2369-2397.

[3] _ Ample twisted face-pairing 3-manifolds, preprint.

[4] _ Heegaard diagrams and surgery descriptions for twisted face-pairing 3-manifolds, Algebr. Geom. Topol. 3 (2003), 235-285.

[5] _ A survey of twisted face-pairing 3-manifolds, preprint.

[6] R. E. Gompf and A. I. Stipsicz, 4-manifolds and Kirby Calculus, Graduate Studies in Mathematics, 20. American Mathematical Society, Providence, RI, 1999.

[7] H. Helling, A. C. Kim, and J. Mennicke, On Fibonacci groups, preprint.

[8] M. Mulazzani, On periodic Takahashi manifolds, Tsukuba J. Math. 25 (2001), no. 2, $229-237$.

[9] D. Rolfsen, Knots and Links, Mathematics Lecture Series, No. 7. Publish or Perish, Inc., Berkeley, Calif., 1976.

[10] B. Ruini and F. Spaggiari, On the structure of Takahashi manifolds, Tsukuba J. Math. 22 (1998), no. 3, 723-739.

[11] M. Takahashi, On the presentations of the fundamental groups of 3-manifolds, Tsukuba J. Math. 13 (1989), no. 1, 175-189.

[12] W. P. Thurston, The geometry and topology of 3-manifold, Lect. Notes, Princeton University, N. J., 1980. 
SUNGBOK HONG

Department of Mathematics

Korea University

SEOUL 136-701, KorEA

E-mail address: shong@korea.ac.kr

KYUNGHWA LEE

Department of Mathematics

Korea University

SEOUl 136-701, KoreA

E-mail address: daepae@korea.ac.kr 